\title{
Conjuntos Dominantes e Dominantes Independentes em Grafos de Petersen Generalizados
}

\author{
A. A. Pereira ${ }^{1}$, C. N. Campos $^{1}$ \\ ${ }^{1}$ Instituto de Computação - Universidade Estadual de Campinas (UNICAMP) \\ Av. Albert Einstein, 1251 - 13083-852 - Campinas - SP - Brazil \\ alessandra.pereira@students.ic.unicamp.br, campos@ic.unicamp.br
}

\begin{abstract}
A dominating set of a graph $G$ is a set $S$ of vertices such that every vertex in $G$ is either in $S$ or is adjacent to a vertex in $S$. An independent dominating set of $G$ is both dominating and independent in $G$. In this work, we study dominating and independent dominating sets of Generalized Petersen graphs.

Resumo. Um conjunto $S \subseteq V(G)$ é um conjunto dominante se todo vértice de $G$ é um elemento de $S$ ou é adjacente a um elemento de $S$. Um conjunto dominante independente de $G$ é, ao mesmo tempo, um conjunto dominante e um conjunto independente em $G$. Neste trabalho, estudamos conjuntos dominantes e conjuntos dominantes independentes dos Grafos de Petersen Generalizados.
\end{abstract}

\section{Introdução}

Seja $G$ um grafo simples, finito e não orientado com conjunto de vértices $V(G)$ e conjunto de arestas $E(G)$. O grau mínimo de $G$ é denotado por $\delta(G)$. Um conjunto $S \subseteq V(G)$ é um conjunto dominante se, para todo $v \in V(G), v \in S$ ou $v$ é adjacente a algum vértice de $S$. Note que $V(G)$ é um conjunto dominante. Desta forma, o desafio é encontrar um conjunto dominante de cardinalidade mínima. A cardinalidade de um menor conjunto dominante de $G$ é chamada de número de dominação e é denotada por $\gamma(G)$. Um conjunto $S \subseteq V(G)$ é independente se todos os seus vértices são dois a dois não adjacentes. Um conjunto dominante independente de $G$ é, ao mesmo tempo, um conjunto dominante e um conjunto independente. O número de dominação independente de $G$, denotado por $i(G)$, é a cardinalidade de um menor conjunto dominante independente de $G$.

Determinar $\gamma(G)$ e $i(G)$ para um grafo arbitrário $G$ são problemas NP-difíceis (Garey e Johnson, 1979), mesmo quando restritos a grafos cúbicos (Liu et al., 2015). Isto estimula a busca por limitantes para $\gamma(G)$ e $i(G)$. Ore (1962) provou que $\gamma(G) \leq|V(G)| / 2$ para $G \operatorname{com} \delta(G) \geq 1$. Blank (1973) e McCuaig e Shepherd (1989) provaram, de maneira independente, que $\gamma(G) \leq 2|V(G)| / 5$ para grafos conexos com $\delta(G) \geq 2$. Reed (1996) mostrou que $\gamma(G) \leq 3|V(G)| / 8$ quando restrito a grafos conexos com $\delta(G) \geq 3$. Todos estes limitantes são justos. Reed conjecturou, ainda, que para grafos cúbicos conexos $\gamma(G) \leq\lceil|V(G)| / 3\rceil$. No entanto, Kostochka e Stodolsky (2005) mostraram que esta conjectura é falsa. De fato, eles exibiram uma classe infinita de grafos cúbicos conexos para os quais $\gamma(G)>\lceil|V(G)| / 3\rceil$. Estes trabalhos estimularam a pesquisa por grafos cúbicos que verificam ou melhoram a conjectura de Reed.

Como todo conjunto dominante independente é um conjunto dominante, segue que $\gamma(G) \leq i(G)$. Decidir se $\gamma(G)=i(G)$ também é um problema NP-completo (Alvarado et al., 2015). De fato, mesmo quando restrita a grafos cúbicos e conexos, a diferença 
$i(G)-\gamma(G)$ pode ser arbitrariamente grande (Žerovnik e Oplerova, 1993). Isto motiva tanto o estudo dos grafos cúbicos com número de dominação limitado pelo valor da conjectura de Reed, como também a determinação do número de dominação independente destes grafos, para avaliar o quão distantes estes dois parâmetros estão. Neste contexto, este trabalho aborda os números de dominação e número de dominação independente dos Grafos de Petersen Generalizados, denotados por $P(l, k)$. Provamos que para $P(l, k)$, $k \in\{1,2,3\}$, o número de dominação é igual ao número de dominação independente, exceto para $P(11,3)$ e para $P(l, 1)$ em que $l \equiv 1(\bmod 4)$.

\section{Resultados}

Watkins (1969) define um Grafo de Petersen Generalizado $P(l, k), k \leq\lfloor(l-1) / 2\rfloor \mathrm{e}$ $l \geq 3$, tal que $V(P(l, k))=X \cup Y, \operatorname{com} X=\left\{x_{i}: 0 \leq i<l\right\}$ e $Y=\left\{y_{i}: 0 \leq i<l\right\}$, e $E(P(l, k))=E_{0} \cup E_{1} \cup E_{2}, \operatorname{com} E_{0}=\left\{x_{i} x_{i+1}: 0 \leq i<l\right\}, E_{1}=\left\{y_{i} y_{i+k}: 0 \leq i<l\right\}$ e $E_{2}=\left\{x_{i} y_{i}: 0 \leq i<l\right\}$, supondo os índices tomados módulo $l$. O número de dominação do $P(l, k)$ tem sido bastante estudado na literatura. No entanto, $\gamma(P(l, k))$ é conhecido em poucos casos. De particular relevância para o nosso trabalho são os resultados obtidos por Ebrahimi et al. (2009), estabelecidos no Teorema 1.

Teorema 1 (Ebrahimi et al. (2009)). Seja $G=P(l, k)$ para $k \in\{1,2,3\}$. Então,

$$
\gamma(G)= \begin{cases}\lfloor l / 2\rfloor+1 & \text { se } k=1 \text { e } l \equiv 1,2 \quad(\bmod 4) \\ \lceil l / 2\rceil & \text { se } k=1 \text { e } l \equiv 0,3 \quad(\bmod 4) \\ \lceil 3 l / 5\rceil & \text { se } k=2 \\ \lceil l / 2\rceil & \text { se } k=3 \text { e } l \equiv 0,1 \quad(\bmod 4) \text { ou } l=11 \\ \lceil l / 2\rceil+1 & \text { se } k=3, l \equiv 2,3 \quad(\bmod 4) \text { e } l \neq 11\end{cases}
$$

Neste trabalho, determinamos o número de dominação independente do $P(l, k)$, $k \in\{1,2,3\}$, e o comparamos com os resultados de Ebrahimi et al. (2009). Em particular, o Teorema 3, com o auxílio do Lema 2, prova que $i(P(l, 1))=\gamma(P(l, 1))$, quando $l \equiv 0,2,3(\bmod 4)$, e $i(P(l, 1))=\gamma(P(l, 1))+1$, quando $l \equiv 1(\bmod 4)$; o Teorema 4 prova que $i(P(l, 2))=\gamma(P(l, 2))$; e o Teorema 5 prova que $i(P(l, 3))=\gamma(P(l, 3))$, quando $l \neq 11$, e $i(P(11,3))=\gamma(P(11,3))+1$.

Lema 2. Se $G=P(l, 1), l \equiv 1(\bmod 4)$, então $i(G) \geq\lfloor l / 2\rfloor+2$.

Teorema 3. Seja $P(l, 1)$ um Grafo de Petersen Generalizado com $l \geq 3$. Então,

$$
i(P(l, 1))= \begin{cases}\lfloor l / 2\rfloor+2 & \text { se } l \equiv 1 \quad(\bmod 4), \\ \lfloor l / 2\rfloor+1 & \text { se } l \equiv 2 \quad(\bmod 4), \\ \lceil l / 2\rceil & \text { se } l \equiv 0,3 \quad(\bmod 4) .\end{cases}
$$

Esboço da demonstração. Seja $G=P(l, 1) \operatorname{com} l=4 t+r, r \in\{0,1,2,3\}$. Note que, pela definição de $k$, se $k=1$, então $l \geq 3$. Seja $S \subseteq V(G)$ tal que $S=A \cup B \cup R$ em que $A=\left\{x_{4 i+1}: 0 \leq i<\lfloor(l+2) / 4\rfloor\right\}, B=\left\{y_{4 i+3}: 0 \leq i<\lfloor l / 4\rfloor\right\}, R=\emptyset$ se $r=0$, $R=\left\{x_{l-1}, y_{0}\right\}$ se $r=1$, e $R=\left\{y_{0}\right\}$ se $r \in\{2,3\}$.

Inicialmente, observe que $S$ é um conjunto independente. Vamos, agora, mostrar que $S$ é um conjunto dominante. Cada $x_{4 i+1} \in A$ domina $x_{4 i}, x_{4 i+1}, y_{4 i+1}$ e $x_{4 i+2}$ 
e cada $y_{4 i+3} \in B$ domina $y_{4 i+2}, y_{4 i+3}, x_{4 i+3}$ e $y_{4 i+4}$. Note que $A=\left\{x_{1}, x_{5}, \ldots, x_{\alpha}\right\}$ e $B=\left\{y_{3}, y_{7}, \ldots, y_{\beta}\right\}$ em que $\alpha=4\lfloor(l+2) / 4\rfloor-3$ e $\beta=4\lfloor l / 4\rfloor-1$. Logo: quando $l \equiv 0(\bmod 4), A \cup B$ domina $V(G)$; quando $l \equiv 1(\bmod 4), A \cup B$ domina $V(G) \backslash\left\{x_{l-1}, y_{0}\right\}$; quando $l \equiv 2(\bmod 4), A \cup B$ domina $V(G) \backslash\left\{y_{0}\right\}$; e, por fim, quando $l \equiv 3(\bmod 4), A \cup B$ domina $V(G) \backslash\left\{y_{l-1}, y_{0}\right\}$. Concluímos que $S$ é um conjunto dominante independente de $G$, dado que $x_{l-1}, y_{0} \in R$ e a aresta $y_{l-1} y_{0} \in E(G)$. Por construção, $|S|=\lceil l / 2\rceil$ se $l \equiv 0,3(\bmod 4),|S|=\lfloor l / 2\rfloor+1$ se $l \equiv 2(\bmod 4)$ e $|S|=\lfloor l / 2\rfloor+2$ se $l \equiv 1(\bmod 4)$. O resultado segue considerando os valores de $\gamma(G)$ determinados por Ebrahimi et al. (2009) e o Lema 2.

Teorema 4. Seja $P(l, 2)$ um Grafo de Petersen Generalizado com $l \geq 5$. Então, $i(P(l, 2))=\lceil 3 l / 5\rceil$.

Esboço da demonstração. Seja $G=P(l, 2), l \geq 5$. Considerando Ebrahimi et al. (2009), segue que $i(G) \geq\lceil 3 l / 5\rceil$. Resta mostrar que $i(G) \leq\lceil 3 l / 5\rceil$. Para isso, construímos um conjunto dominante independente $S$ com esta cardinalidade.

Seja $l=5 t+r, r \in\{0,1,2,3,4\}$. Particione $V(G)$ em $t$ partes $B^{0}, B^{2}, \ldots, B^{t-1}$ tais que $B^{i}=\left\{x_{5 i+j}, y_{5 i+j}: 0 \leq j \leq 4\right\}$, e uma parte adicional $B^{t}=\left\{x_{l-r+j}, y_{l-r+j}\right.$ : $0 \leq j<r\}$ se $r \neq 0$ (considere $B^{t}=\emptyset$ se $r=0$ ). Note que $\left|B^{i}\right|=10$ quando $0 \leq i<t$ e $\left|B^{t}\right|=2 r$. Para $0 \leq i<t$, seja $D^{i}=\left\{x_{5 i+1}, y_{5 i+2}, x_{5 i+3}\right\}$. A Figura 1 ilustra $B^{i}$ e $D^{i}$. Note que $D^{i}$ domina todos os vértices de $B^{i}$ e que seus vértices não são adjacentes entre si e nem a vértices de $B^{j}, j \neq i$. Desta forma, $S^{0}=\cup_{i=0}^{t-1} D^{i}$ domina $G\left[V(G) \backslash B^{t}\right]$.

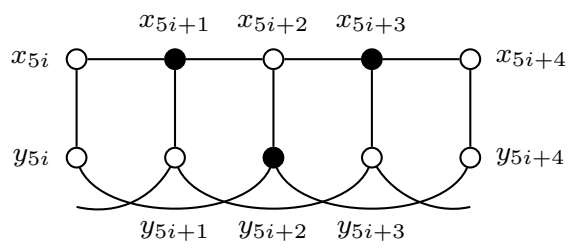

Figura 1. Bloco $B^{i}$. Vértices de $D^{i}$ representados em preto.

Para $r=0, G=G\left[\cup_{i=0}^{t-1} B^{i}\right]$. Logo, $S^{0}$ domina $V(G)$ e $S^{0}=\lceil 3 l / 5\rceil$. Resta, analisar $B^{t}, r \in\{1,2,3,4\}$. Sejam $S^{1}=\left\{y_{l-1}\right\}, S^{2}=\left\{x_{l-2}\right\}, S^{3}=\emptyset$ e $S^{4}=\left\{y_{l-4}\right\}$. Como $B^{t}=\left\{x_{l-r+j}, y_{l-r+j}: 0 \leq j<r\right\}$, concluímos que $S=\cup_{i=0}^{r} S^{i}$ é um conjunto dominante independente de $G$. Ademais, $|S|=3 t+1$ se $r=1,|S|=3 t+2$ se $r \in\{2,3\}$ e $|S|=3 t+3$ se $r=4$. Em todos os casos, $|S|=\lceil 3 l / 5\rceil$ e o resultado segue.

Teorema 5. Seja $P(l, 3)$ um Grafo de Petersen Generalizado com $l \geq 7$. Então,

$$
i(P(l, 3))=\left\{\begin{array}{lll}
\lceil l / 2\rceil & \text { se } l \equiv 0,1 \quad(\bmod 4) ; \\
\lceil l / 2\rceil+1 & \text { se } l \equiv 2,3 \quad(\bmod 4) .
\end{array}\right.
$$

Esboço da demonstração. Seja $G=P(l, 3), l \geq 7$. Como nos casos anteriores, $i(G) \geq \gamma(G)$, com $\gamma(G)$ determinado por Ebrahimi et al. (2009).

Considere, inicialmente, $G=P(11,3)$. É possível mostrar que todo conjunto dominante mínimo neste grafo possui um par de vértices adjacentes (Ebrahimi et al., 2009). Logo, $i(G)>\gamma(G)=6$. A figura ao lado exibe um conjunto dominante independente de $G$ com cardinalidade sete. Logo, $i(G)=7=\lceil l / 2\rceil+1$ e o resultado segue.

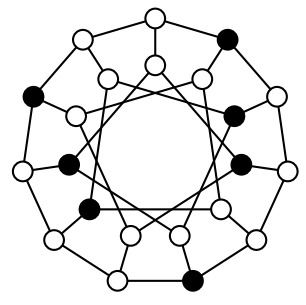


Suponha, agora, $l \neq 11$ com $l=4 t+r, r \in\{0,1,2,3\}$. Seja $S \subseteq V(G)$ tal que $S=A \cup B \cup R$ em que $A=\left\{x_{4 i+1}: 0 \leq i<\lfloor(l+1) / 4\rfloor\right\}$, $B=\left\{y_{4 i+3}: 0 \leq i<\lfloor l / 4\rfloor\right\}, R=\emptyset$ se $r=0, R=\left\{y_{l-1}\right\}$ se $r=1, R=\left\{x_{l-2}, y_{l-1}\right\}$ se $r=2$ e $R=\left\{y_{2}, y_{l-3}\right\}$ se $r=3$.

Inicialmente, observe que $S$ é um conjunto independente. Vamos, agora, mostrar que $S$ é um conjunto dominante. Cada $x_{4 i+1} \in A$ domina $x_{4 i}, x_{4 i+1}, y_{4 i+1}$ e $x_{4 i+2}$, e cada $y_{4 i+3} \in B$ domina $y_{4 i}, y_{4 i+3}, x_{4 i+3}$ e $y_{4 i+6}$. Note que $A=\left\{x_{1}, x_{5}, \ldots, x_{\alpha}\right\}$ e $B=\left\{y_{3}, y_{7}, \ldots, y_{\beta}\right\}$ em que $\alpha=4\lfloor(l+1) / 4\rfloor-3$ e $\beta=4\lfloor l / 4\rfloor-1$. Logo: quando $l \equiv 0(\bmod 4), A \cup B$ dominam $V(G) ;$ quando $l \equiv 1(\bmod 4), A \cup B$ domina $V(G) \backslash\left\{y_{2}, x_{l-1}, y_{l-1}\right\} ;$ quando $l \equiv 2(\bmod 4), A \cup B$ domina $V(G) \backslash$ $\left\{y_{2}, x_{l-2}, x_{l-1}, y_{l-2}, y_{l-1}\right\}$; e, por fim, quando $l \equiv 3(\bmod 4), A \cup B$ domina $V(G) \backslash\left\{y_{2}, y_{l-3}\right\}$. O resultado segue considerando que os vértices não dominados por $A \cup B$ ou estão em $R$ ou são adjacentes a um vértice de $R$.

Neste trabalho, estudamos o número de dominação e o número de dominação independente dos Grafos de Petersen Generalizados $P(l, k), k \in\{1,2,3\}$. A partir dos resultados obtidos, conjecturamos que a diferença $i(P(l, k))-\gamma(P(l, k))$ esteja limitada a um. A análise preliminar dos casos $k=4$ e $k=5$ verifica esta conjectura. Entretanto, os casos em que a diferença ocorre ainda não estão bem caracterizados. Nossos estudos estão agora focados nesta conjectura e na busca por uma propriedade que determine quais são os grafos para os quais $i(P(l, k)) \neq \gamma(P(l, k))$.

\section{Referências}

Alvarado, J. D., Dantas, S., e Rautenbach, D. (2015). Complexity of comparing the domination number to the independent domination, connected domination, and paired domination numbers. Matemática Contemporânea, 44(1):1-8.

Blank, M. (1973). An estimate of the external stability number of a graph without suspended vertices. Prikl. Math. i Programmirovanie, 10:3-11.

Ebrahimi, B. J., Jahanbakht, N., e Mahmoodian, E. S. (2009). Vertex domination of generalized petersen graphs. Discrete mathematics, 309(13):4355-4361.

Garey, M. R. e Johnson, D. S. (1979). Computers and Intractability: A Guide to the Theory of NP-Completeness. Freeman, New York, US.

Kostochka, A. V. e Stodolsky, B. Y. (2005). On domination in connected cubic graphs. Discrete mathematics, 304(1-3):45-50.

Liu, C. H., Poon, S. H., e Lin, J. Y. (2015). Independent dominating set problem revisited. Theoretical Computer Science, 562:1-22.

McCuaig, W. e Shepherd, B. (1989). Domination in graphs with minimum degree two. Journal of Graph Theory, 13(6):749-762.

Ore, O. (1962). Theory of Graphs. American Mathematical Society, Providence, US.

Reed, B. (1996). Paths, stars and the number three. Combinatorics, Probability and Computing, 5(3):277-295.

Žerovnik, J. e Oplerova, J. (1993). A counterexample to conjecture of Barefoot, Harary, and Jones. Graphs and Combinatorics, 9(2-4):205-207.

Watkins, M. E. (1969). A theorem on Tait colorings with an application to the generalized Petersen graphs. Journal of Combinatorial Theory, 6(2):152-164. 